\title{
Doublet identification in single-cell sequencing data using
}

\section{scDblFinder [version 1; peer review: 1 approved, 1 approved}

\section{with reservations]}

\author{
Pierre-Luc Germain (D1-3, Aaron Lun4, Will Macnair5, Mark D. Robinson (D1,3 \\ ${ }^{1}$ DMLS Lab of Statistical Bioinformatics, University of Zürich, Zürich, 805, Switzerland \\ ${ }^{2}$ D-HEST Institute for Neuroscience, ETH Zürich, Zürich, Switzerland \\ ${ }^{3}$ Swiss Institute of Bioinformatics, University of Zürich, Zürich, Switzerland \\ ${ }^{4}$ Genentech Inc., South San Francisco, CA, USA \\ ${ }^{5}$ Pharma Research and Early Development, Neuroscience, Ophthalmology and Rare Diseases, F. Hoffmann-LaRoche Ltd, Basel, \\ Switzerland
}

V1 First published: $28 \operatorname{Sep} 2021,10: 979$

https://doi.org/10.12688/f1000research.73600.1

Latest published: 16 May 2022, 10:979

https://doi.org/10.12688/f1000research.73600.2

\section{Abstract}

Doublets are prevalent in single-cell sequencing data and can lead to artifactual findings. A number of strategies have therefore been proposed to detect them. Building on the strengths of existing approaches, we developed scDblFinder, a fast, flexible and accurate Bioconductor-based doublet detection method. Here we present the method, justify its design choices, demonstrate its performance on both single-cell RNA and accessibility sequencing data, and provide some observations on doublet formation, detection, and enrichment analysis. Even in complex datasets, scDb/Finder can accurately identify most heterotypic doublets, and was already found by an independent benchmark to outcompete alternatives.

Keywords

single-cell sequencing, doublets, multiplets, filtering

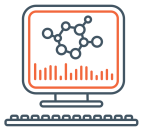

This article is included in the Bioinformatics gateway.

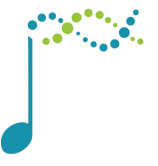

This article is included in the Bioconductor gateway.

\section{Open Peer Review}

Approval Status

1 2

version 2

(revision)

16 May 2022

version 1

28 Sep 2021

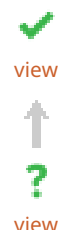

view

1. Zev J. Gartner ID, University of California,

San Francisco, San Francisco, USA

Chris McGinnis, University of California, San

Francisco, USA

2. Dennis Kostka ID, University of Pittsburgh School of Medicine, Pittsburgh, USA

Hannah Schriever, University of Pittsburgh

School of Medicine, Pittsburgh, USA

Joint CMU-Pitt Ph.D. Program in

Computational Biology, Pittsburgh, USA
Any reports and responses or comments on the article can be found at the end of the article. 
Corresponding authors: Pierre-Luc Germain (pierre-luc.germain@uzh.ch), Mark D. Robinson (mark.robinson@mls.uzh.ch)

Author roles: Germain PL: Conceptualization, Formal Analysis, Methodology, Software, Writing - Original Draft Preparation; Lun A: Software, Writing - Review \& Editing; Macnair W: Methodology, Writing - Review \& Editing; Robinson MD: Conceptualization, Funding Acquisition, Supervision, Writing - Review \& Editing

Competing interests: No competing interests were disclosed.

Grant information: This work was supported by the Swiss National Science Foundation (grant number 310030_175841).

The funders had no role in study design, data collection and analysis, decision to publish, or preparation of the manuscript.

Copyright: ( 2021 Germain PL et al. This is an open access article distributed under the terms of the Creative Commons Attribution License, which permits unrestricted use, distribution, and reproduction in any medium, provided the original work is properly cited.

How to cite this article: Germain PL, Lun A, Macnair W and Robinson MD. Doublet identification in single-cell sequencing data using scDblFinder [version 1; peer review: 1 approved, 1 approved with reservations] F1000Research 2021, 10:979

https://doi.org/10.12688/f1000research.73600.1

First published: 28 Sep 2021, 10:979 https://doi.org/10.12688/f1000research.73600.1 


\section{Introduction}

High-throughput single-cell sequencing, in particular single-cell/nucleus RNA-sequencing (scRNAseq), has provided an unprecedented resolution on biological phenomena. A particularly popular approach uses oil droplets or wells to isolate single cells along with barcoded beads. Depending on the cell density loaded, a proportion of reaction volumes (i.e. droplets or wells) will capture more than one cell, forming 'doublets' (or 'multiplets'), i.e. two or more cells captured by a single reaction volume and thus sequenced as a single-cell artifact. The proportion of doublets has been shown to be proportional to the number of cells captured (Bloom 2018; Kang et al. 2018). It is therefore at present common in singlecell experiments to have $10-20 \%$ doublets, making accurate doublet detection critical.

'Homotypic' doublets, which are formed by cells of the same type (i.e. similar transcriptional state), are very difficult to identify on the basis of their transcriptome alone (McGinnis, Murrow, and Gartner 2019). They are also, however, relatively innocuous for most purposes, as they appear highly similar to singlets. 'Heterotypic' doublets (formed by cells of distinct transcriptional states), instead, can appear as an artifactual novel cell type and disrupt downstream analyses (Germain, Sonrel, and Robinson 2020).

Experimental methods have been devised for detecting doublets in multiplexed samples, using barcodes (Stoeckius et al. 2018) or genotypes (e.g. single-nucleotide polymorphisms) to identify droplets containing material from more than one sample (Kang et al. 2018). While evidently useful, these identify only a fraction of the doublets, and fail to detect doublets formed by cells from the same sample, including heterotypic doublets.

A number of computational approaches have therefore been developed to identify doublets on the basis of their transcriptional profile (McGinnis, Murrow, and Gartner 2019; DePasquale et al. 2019; Wolock, Lopez, and Klein 2019; Bais and Kostka 2020; Bernstein et al. 2020). Most of these approaches rely on the generation of artificial doublets by summing or averaging real cells, and score the similarity between them and the real cells. For example, DoubletFinder generates a $k$-nearest neighbor $(\mathrm{kNN})$ graph on the union of real cells and artificial doublets, and estimates the density of artificial doublets in the neighborhood of each cell (McGinnis, Murrow, and Gartner 2019). In a similar fashion, one of the methods proposed by Bais and Kostka (2020), bcds, generates artificial doublets and trains a classifier to distinguish them from real cells. Real cells that are classified with artificial doublets are then called as doublets. Finally, another strategy proposed by Bais and Kostka (2020) is a coexpression score, cxds, which flags cells that co-express a number of genes that otherwise tend to be mutually exclusive across cells.

Xi and Li (2021a) recently reported a benchmark of computational doublet detection methods, using both simulations and real datasets with genotype-based true doublets. Interestingly, despite several new publications, the benchmark identified the oldest method, DoubletFinder (McGinnis, Murrow, and Gartner 2019), as the most accurate. However, another important observation from the benchmark was that no single method was systematically the best across all datasets, highlighting the necessity to test and benchmark methods across a variety of datasets, and suggesting that some strategies might have advantages and disadvantages across situations.

Here, we present the scDblFinder package, building on the extensive single-cell Bioconductor methods and infrastructures (Amezquita et al. 2019) and implementing a number of doublet detection approaches. In particular, the scDblFinder method integrates insights from previous approaches and novel improvements to generate fast, flexible and robust doublet prediction. scDblFinder was independently tested by $\mathrm{Xi}$ and $\mathrm{Li}$ in the protocol extension to their initial benchmark and was found to have the best overall performance (Xi and Li 2021b).

\section{Methods}

scDblFinder implementation

Figure 1 gives an overview of the scDblFinder method.

As a first step, the dataset is reduced to its top most expressed features (1000 by default); if the cluster-based approach is used, the top features per cluster are instead selected.

If using the cluster-based approach (and not manually specifying the clusters), a fast clustering is performed (see Fast clustering). Artificial doublets are then created by combining cells of different clusters, proportional to the cluster sizes. In explicitly concentrating on inter-cluster doublets, we do not attempt to identify homotypic doublets, which are virtually unidentifiable and relatively innocuous anyway. In doing so, we reduce the necessary number of artificial doublets (since no artificial doublet is 'lost' modeling homotypic doublets), and prevent the classifier from being trained to recognize cells that are indistinguishable from singlets (and would therefore call singlets as doublets). An alternative strategy, also available through scDblFinder, is to generate fully random artificial doublets, and use the iterative procedure (see below) 


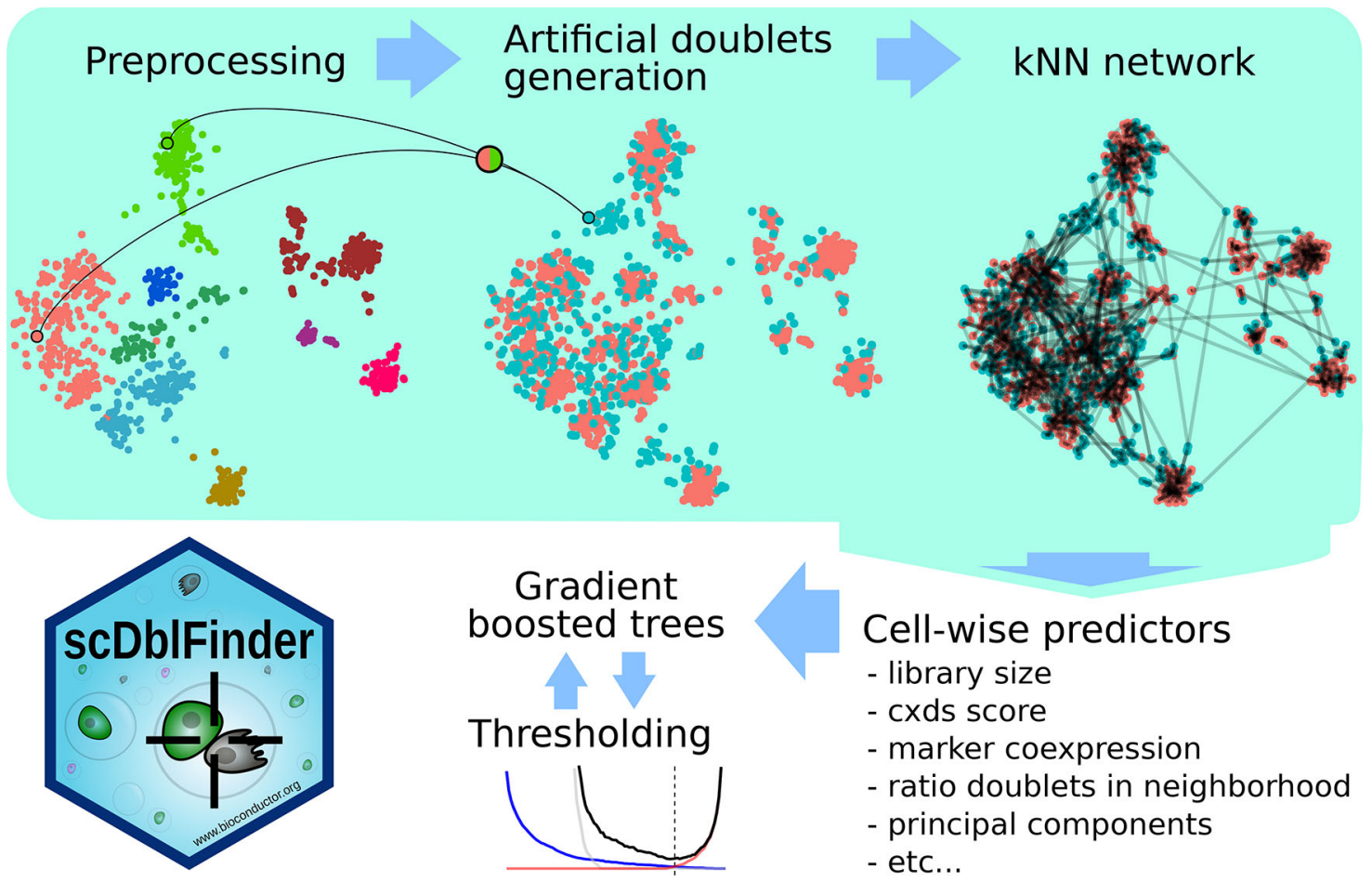

Figure 1. Overview of the scDbIFinder method.

to exclude unidentifiable artificial doublets from the training. In practice, the two approaches have comparable performances (see below), and they can also be combined.

Dimension reduction is then performed on the union of real cells and artificial doublets, and a nearest neighbor network is generated. The network is then used to estimate a number of characteristics for each cell, in particular the proportion of artificial doublets among the nearest neighbors. Rather than selecting a specific neighborhood size, the ratio is calculated at different values of $k$, creating multiple predictors that will be used by the classifier. A distance-weighted ratio is also included. Further cell-level predictors are added, including: projections on principal components; library size; and co-expression scores (based on a variation of Bais and Kostka 2020). scDblFinder then trains gradient boosted trees to distinguish, based on these features, artificial doublets from real cells. Finally, a thresholding procedure decides the score at which to call a cell by simultaneously minimizing the misclassification rate and the expected doublet rate (see Thresholding).

A key problem with classifier-based approaches is that some of the real cells are mislabeled, in the sense that they are in fact doublets labeled as singlets. These can mislead the classifier. For this reason, classification and thresholding are performed in an iterative fashion: at each round, the real cells identified as doublets are removed from the training data for the next round.

Using the benchmark datasets from Xi and Li (2021a), we next optimized a number of parameters in the procedure, notably regarding features to include and hyperparameters, so as to provide robust default parameters (see Germain 2021b, Figures 1-6). Some features, such as the distance to the nearest doublet or whether the nearest neighbor is an artificial doublet, had a negative impact on performance (see Germain 2021b, Figure 1), presumably because it led to over-fitting. Indeed, because artificial doublet creation can only approximate real doublets, a risk of classifier-based approaches is that the exact classification problem on which they are trained, namely distinguishing artificial doublets from real cells, slightly differs from the real problem on which they are expected to function (distinguishing real doublets from real singlets). To test the hypothesis that this can lead to overfitting, we used scDblFinder without the dimensional reduction and $\mathrm{kNN}$ steps, which arguably involve a loss of information, and trained the classifier directly on the expression of the selected genes. This resulted in a reduction in area under the precision and recall curve (AUPRC) in real datasets (see Germain 2021b, Figure 2; see also Figure 4). Finally, in line with a discrepancy between the trained and real problems, we observed that the variable importance calculated during training (see Germain 2021b, Figure 3) did not necessarily match that of the variable drop experiments (see Germain 2021b, Figure 1). 


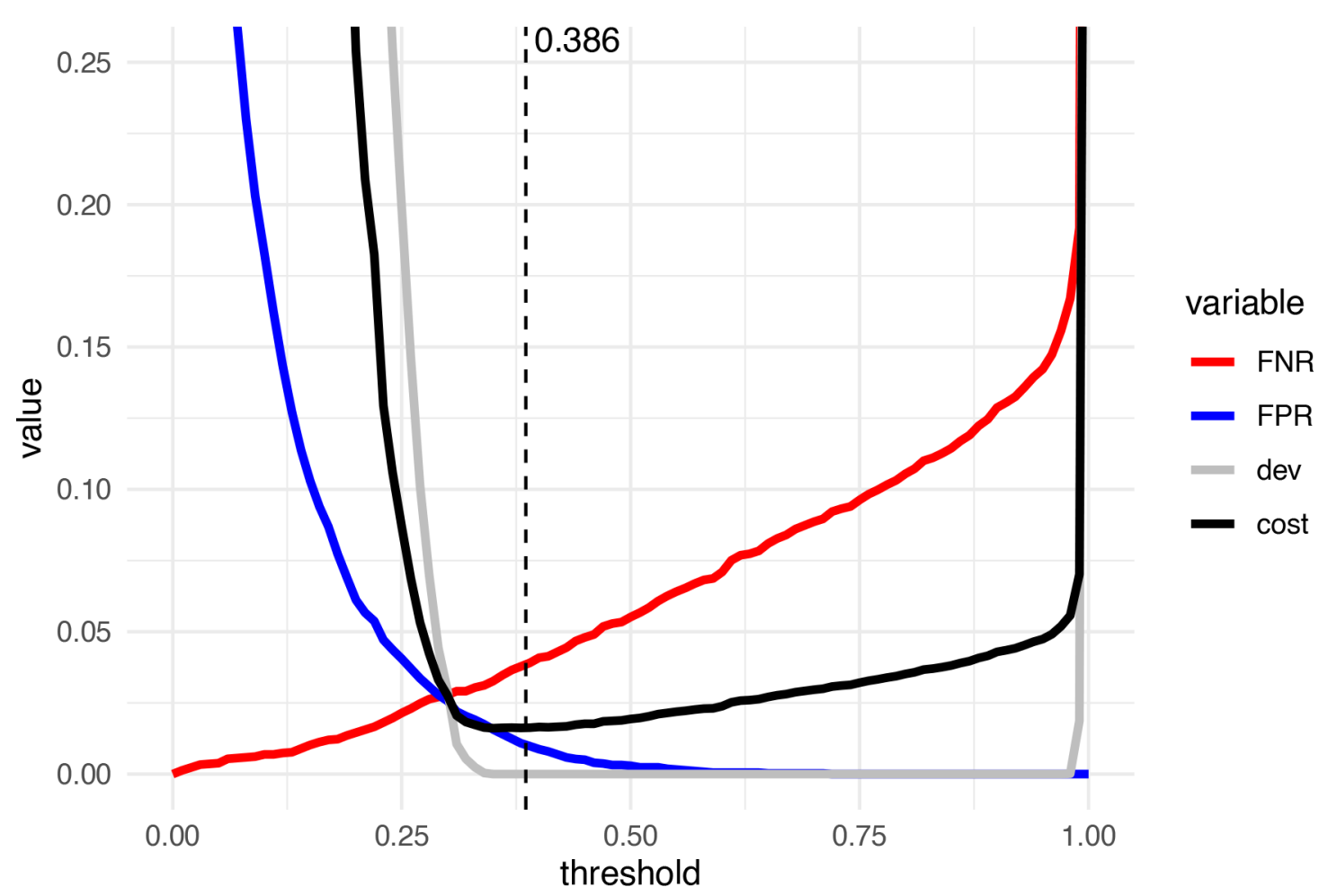

Figure 2. Illustration of the cost function to be minimized for thresholding. Plotted are the false negative rate (FNR; the rate of misclassified artificial doublets), the false positive rate (FPR; the proportion of real cells classified as doublets), the squared proportion deviation from the expected doublet rate (denoted 'dev'), and the integrated cost function to be minimized (mean of the previous). The dashed line indicates the threshold called.

We finally optimized hyperparameters (see Germain 2021b, Figure 4) as well as the number of iterations (see Germain 2021b, Figure 5), finding that a relatively low number of iterations (2-3) was sufficient.

\section{Fast clustering}

Irlba-based singular value decomposition is first run using the scater package, and a kNN network is generated using the Annoy approximation implemented in BiocSingular. Louvain clustering is then used on the graph. If the dataset is sufficiently large (>1000 cells), a first rapid k-means clustering (using the mbkmeans package) is used to generate a large number of meta-cells, which are then clustered using the graph-based approach, propagating clusters back to the cells themselves.

\section{Thresholding}

Unless manually given, the expected number of doublets $\left(e\right.$ ) is specified by $e=n^{2} / 10^{-5}$ (where $n$ is the number of cells captured). This is then restricted to heterotypic doublets using random expectation from cluster sizes or, if not using the cluster-based approach, using the proportion of artificial doublets misidentified. The doublet rate is accompanied by an uncertainty interval (dbr.sd parameter), and the deviation from the expected doublet number for threshold $t$ is then calculated as

$$
\text { deviation }_{t}= \begin{cases}0 & \text { if }\left(o_{t} \geq e_{\text {low }} \wedge o_{t} \leq e_{\text {high }}\right) \\ 2 \cdot \frac{\min \left(\left|o_{t}-e_{\text {low }}\right|,\left|o_{t}-e_{\text {high }}\right|\right)}{e_{\text {low }}+e_{\text {high }}} & \text { otherwise }\end{cases}
$$

where $o_{t}$ represents the number of real cells classified as doublets at threshold $t$, and $e_{\text {low }}$ and $e_{\text {high }}$ represent, respectively, the lower and higher bounds of the expected number of heterotypic doublets in the dataset (based on the given or estimated doublet rate the dbr.sd parameter). The cost function being minimized is then simply given by cost $_{t}=$ $F N R_{t}+F P R_{t}+$ deviation $_{t}^{2}$, where the false negative rate $\left(F N R_{t}\right)$ represents the proportion of artificial doublets 
A
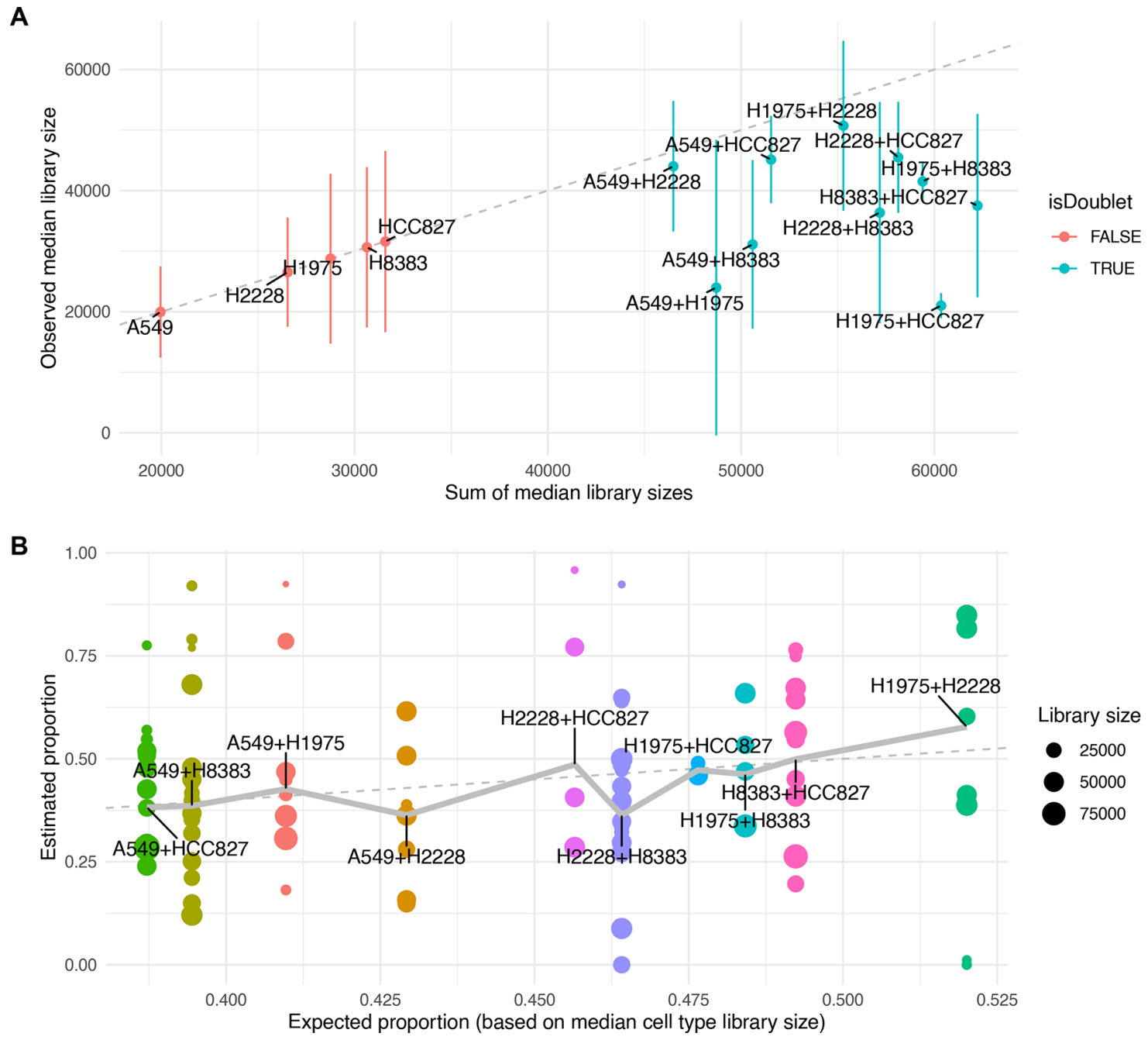

Figure 3. Characterization of real doublets. A: Observed median (and +/- one median absolute deviation in) library sizes per cell type against additive expectation for single cell and doublet types in a real dataset. The dashed line indicates the diagonal. B: Relative contribution of composing cell types in real doublets (each point represents a doublet) plotted against the expected relative contributions (based on the ratio between the median library sizes of the composing cell types). Values indicate the relative contribution of one of the two cell types to the doublet's transcriptome. The dashed line indicates the diagonal, and the thick line indicates the weighted mean per doublet type.

misclassified as singlets at threshold $t$, and the false positive rate $\left(F P R_{t}\right)$ represents the proportion of real cells classified as doublets. This is illustrated in Figure 2.

Since this is performed in an iterative fashion, the FPR is calculated ignoring cells which were called as doublets in the previous round.

Doublet enrichment analysis

Cluster stickiness

Cluster 'stickiness' can be evaluated by fitting a single generalized linear model on the observed abundance of doublets of each origin, in the following way:

$$
\log \left(\text { observed }_{i}+0.1\right)=\log \left(e_{i}\right)+\beta_{z} \cdot \log \left(\text { difficulty }_{i}\right)+\beta_{a} a_{i}+\beta_{b} b_{i}+\beta_{c} c_{i}+\ldots+\epsilon_{i},
$$

where observed $_{i}$ and $e_{i}$ represent the numbers of doublets formed by specific combination $i$ of clusters which are respectively observed or expected from random combinations, and $a_{i}, b_{i}$ and $c_{i}$ (etc) indicate whether or not $(0 / 1)$ the doublet involves each cluster. 


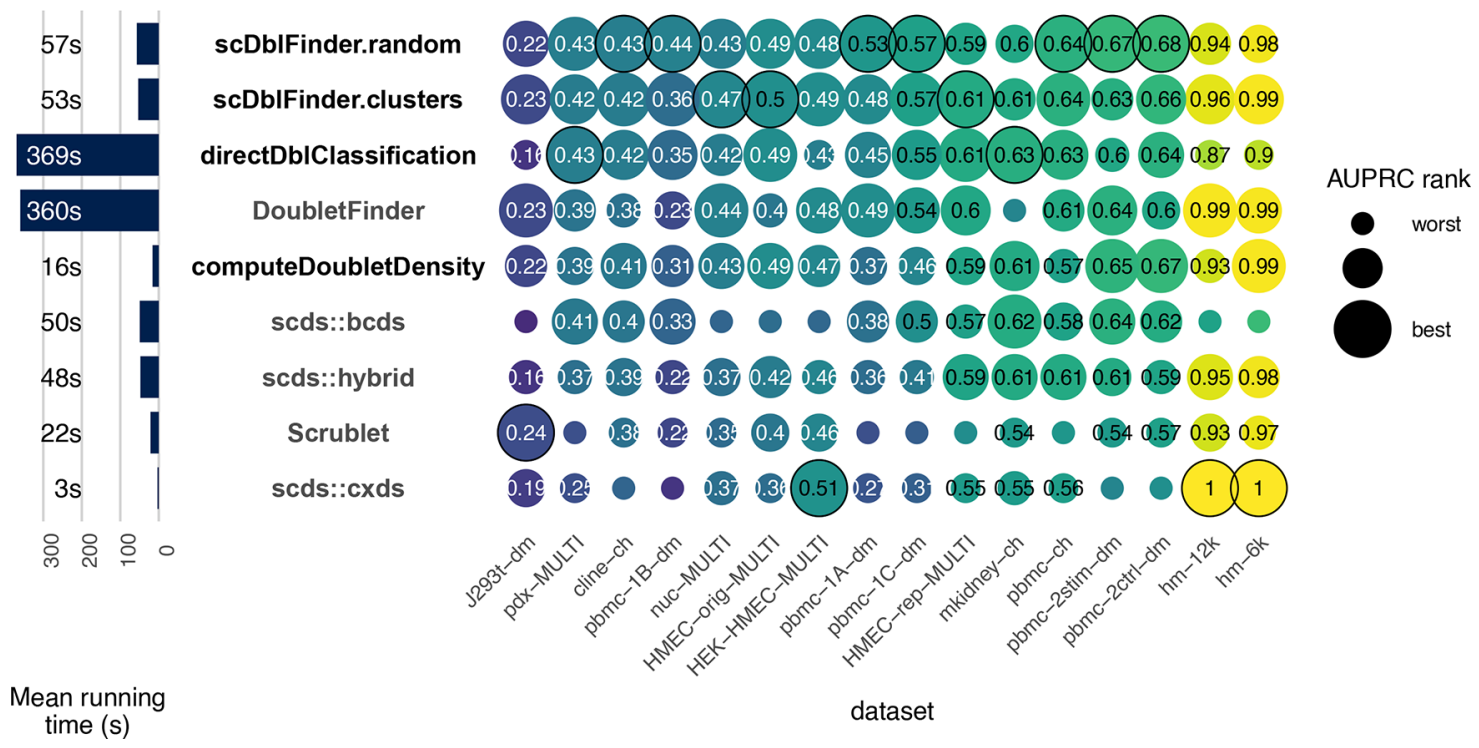

Figure 4. Benchmark. Accuracy (area under the precision and recall curve) of doublet identification using alternative methods across 16 benchmark datasets. The size of the dots indicate the relative ranking for the dataset, and the numbers indicate the actual area under the (PR) curve. For each dataset, the top method is circled in black. Methods in bold are available through the scDblFinder package.

A

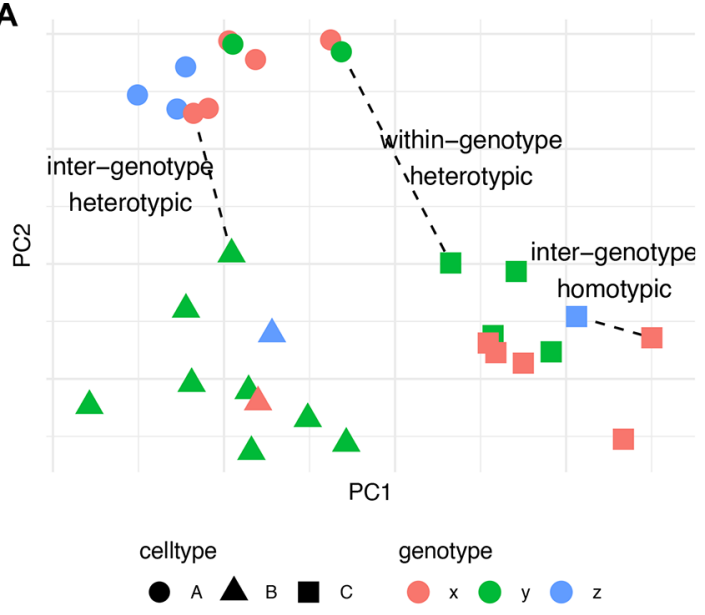

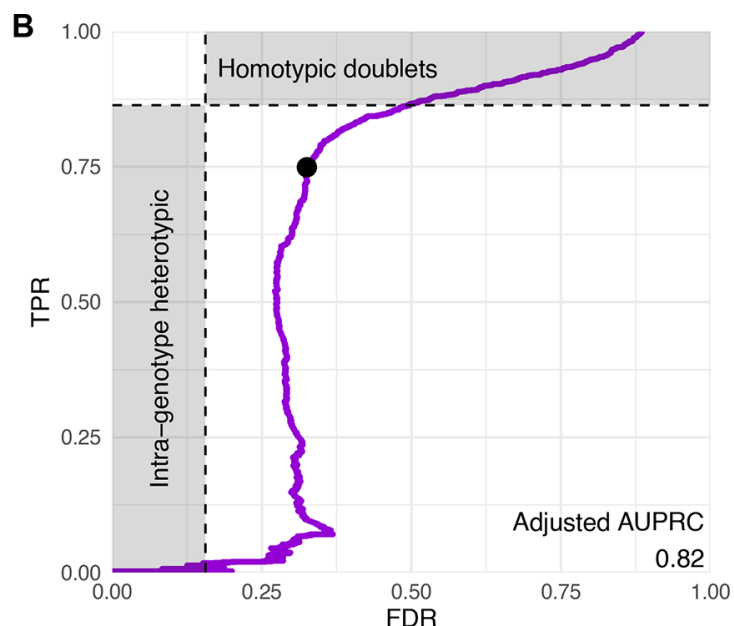

Figure 5. Doublet types and real accuracy of heterotypic doublet identification. A: Schematic (toy data) representing the different types of doublets. Within-genotype heterotypic doublets will wrongly be labeled as false positives, and inter-genotype homotypic will be labeled as false negatives. B: Adjusted PR curve for an example sample (GSM2560248). The two shaded areas represent the expected proportion of within-genotype heterotypic doublets (i.e. wrongly labeled as singlets in the truth) and inter-genotype homotypic doublets, respectively.

Because some doublets are easier to identify than others, some deviation from their expected abundance is typically observed. For this reason, a difficulty ${ }_{i}$ term is optionally included, indicating the difficulty in identifying doublets of origin $i$, estimated from the misclassification of scDblFinder's artificial doublets of that origin (by default, the term is included if at least seven clusters). A $\beta_{a}$ significantly different from zero, then, indicates that cluster $a$ forms more or less doublets than expected - if positive, it indicates cluster 'stickiness.'

For the (quasi-)binomial distributions, logit was used instead of log transformation, and the mean of observed and expected counts was used as observational weights. 

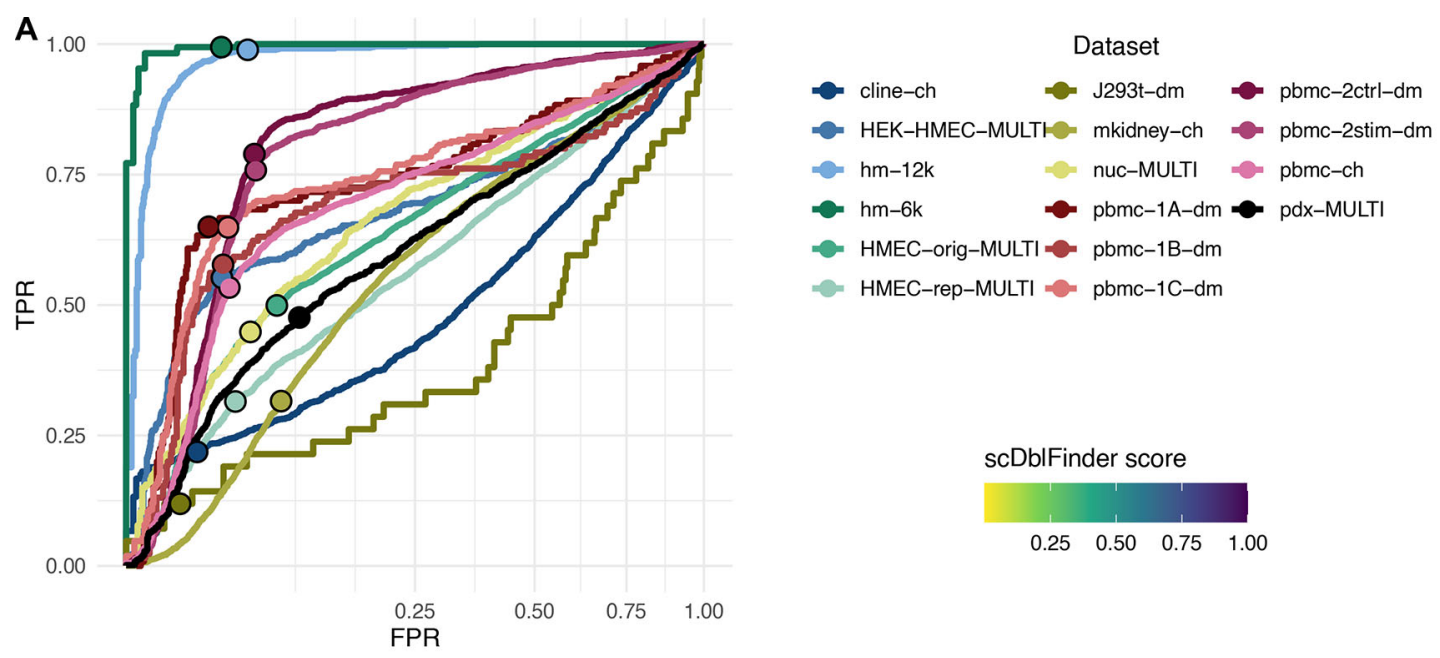

B Using scDblFinder thresholds

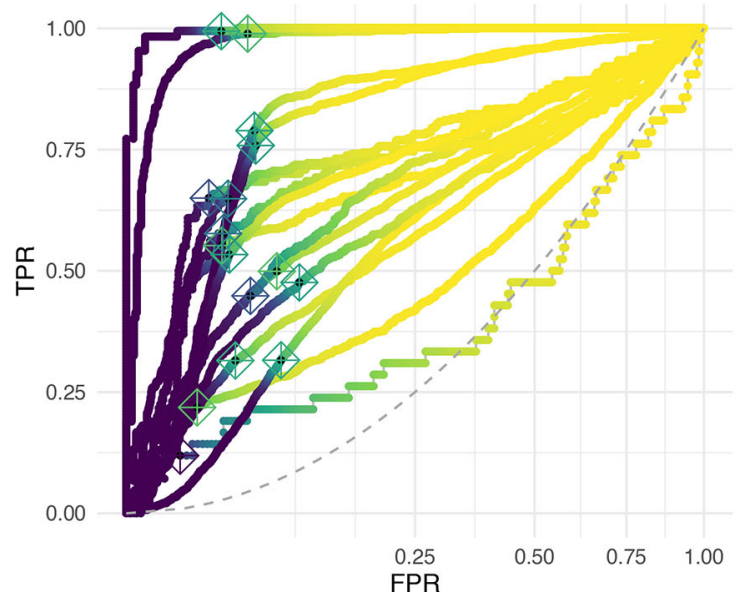

\section{Using expected \# of heterotypic doublets}

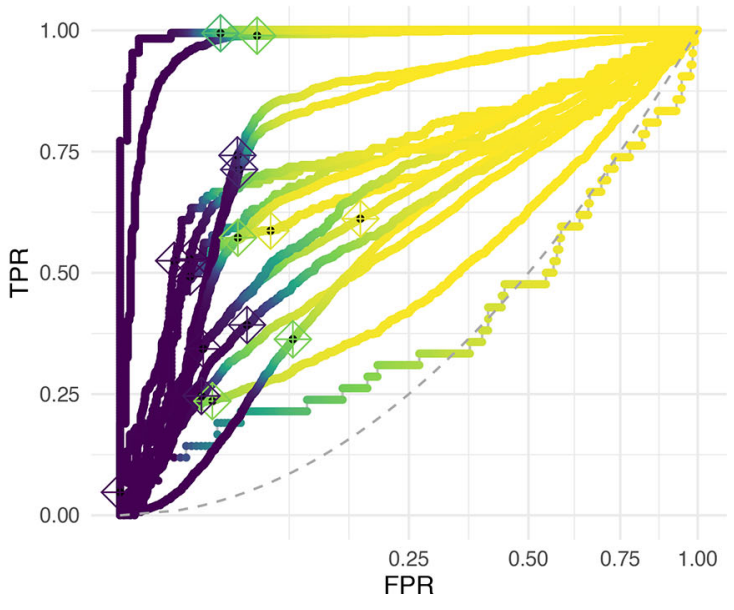

Figure 6. Thresholding. Receiver-operator characteristic (ROC) curves (with square-root transformation on the $x$ axis) of the different benchmark datasets. In $B-C$, the colors indicate the scDblFinder doublet scores, and the crosses indicate the thresholds established through the thresholding method (B) or by taking the expected number of heterotypic doublets (C).

\section{Enrichment for specific combinations}

To account for the different identification difficulty across doublet types, we first fit the following global negative binomial model:

$$
\log \left(\text { observed }_{i}\right)=\alpha+\log \left(e_{i}\right)+\beta \cdot \log \left(\text { difficulty }_{i}\right)
$$

The fitted values are then considered the expected abundance, and the probability of each double type count given this expectation is calculated using either underlying distributions (for the negative binomial, the global over-dispersion parameter calculated in the first step is used).

\section{Direct classification}

The direct classification approach is implemented in the directDblClassification function of the package. It uses the same doublet generation, thresholding and iterative learning procedures as scDblFinder, but trains directly on the normalized expression matrix of real and artificial cells instead of $\mathrm{kNN}$-based features. The hyperparameters were the same except for the maximum tree depth, which was increased to six to account for the increased complexity of the predictors.

Feature aggregation

For feature aggregation, scDblFinder first normalizes the counts using the Term Frequency - Inverse Document Frequency (TF-IDF) normalization, as implemented in Stuart et al. (2019). Principal component analysis (PCA) is then 


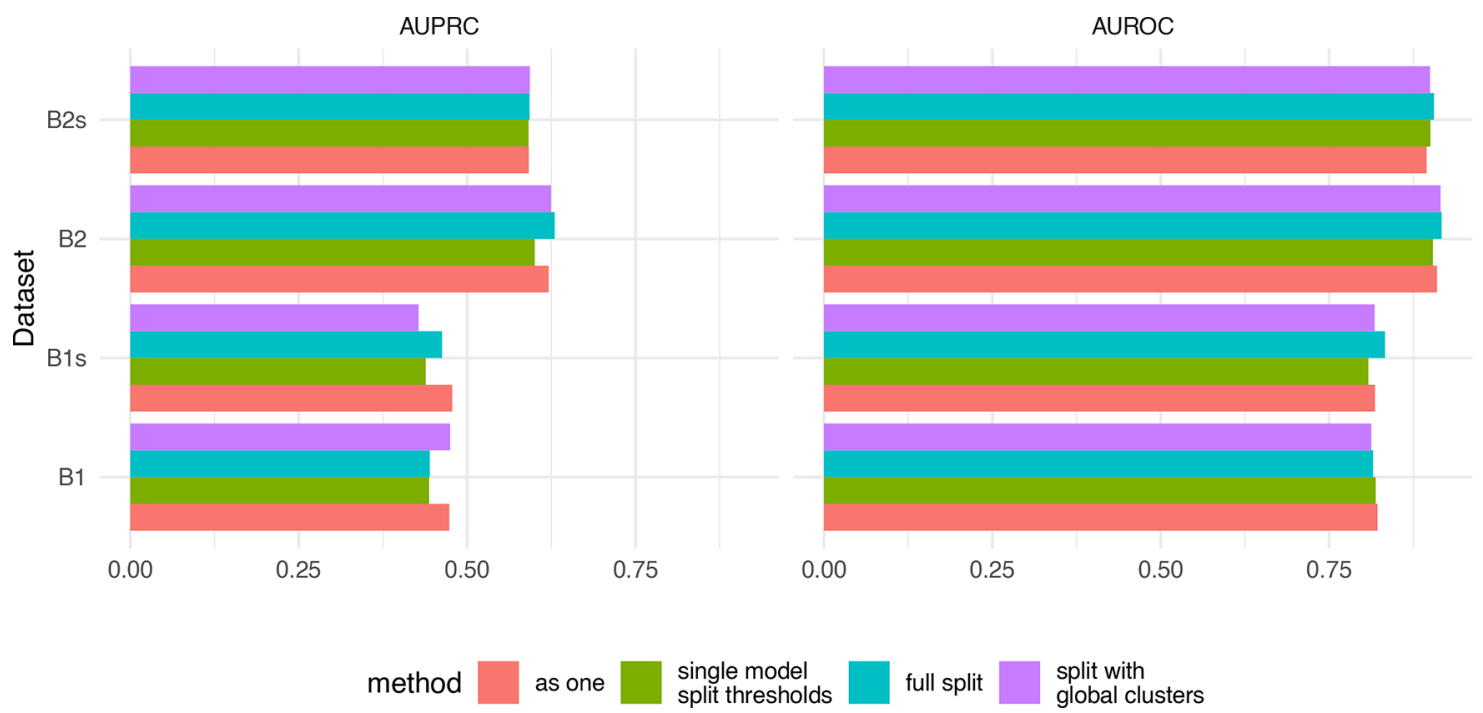

Figure 7. Comparison of four multi-sample strategies. B1 and B2 the two batches from dataset GSE96583, and contain 3 and 2 captures, respectively. The datasets with the suffix 's' are versions downsampled to 30\%. Using doublet detection on each capture separately (full split) was generally comparable to treating the captures as one (and adjusting the doublet rate).

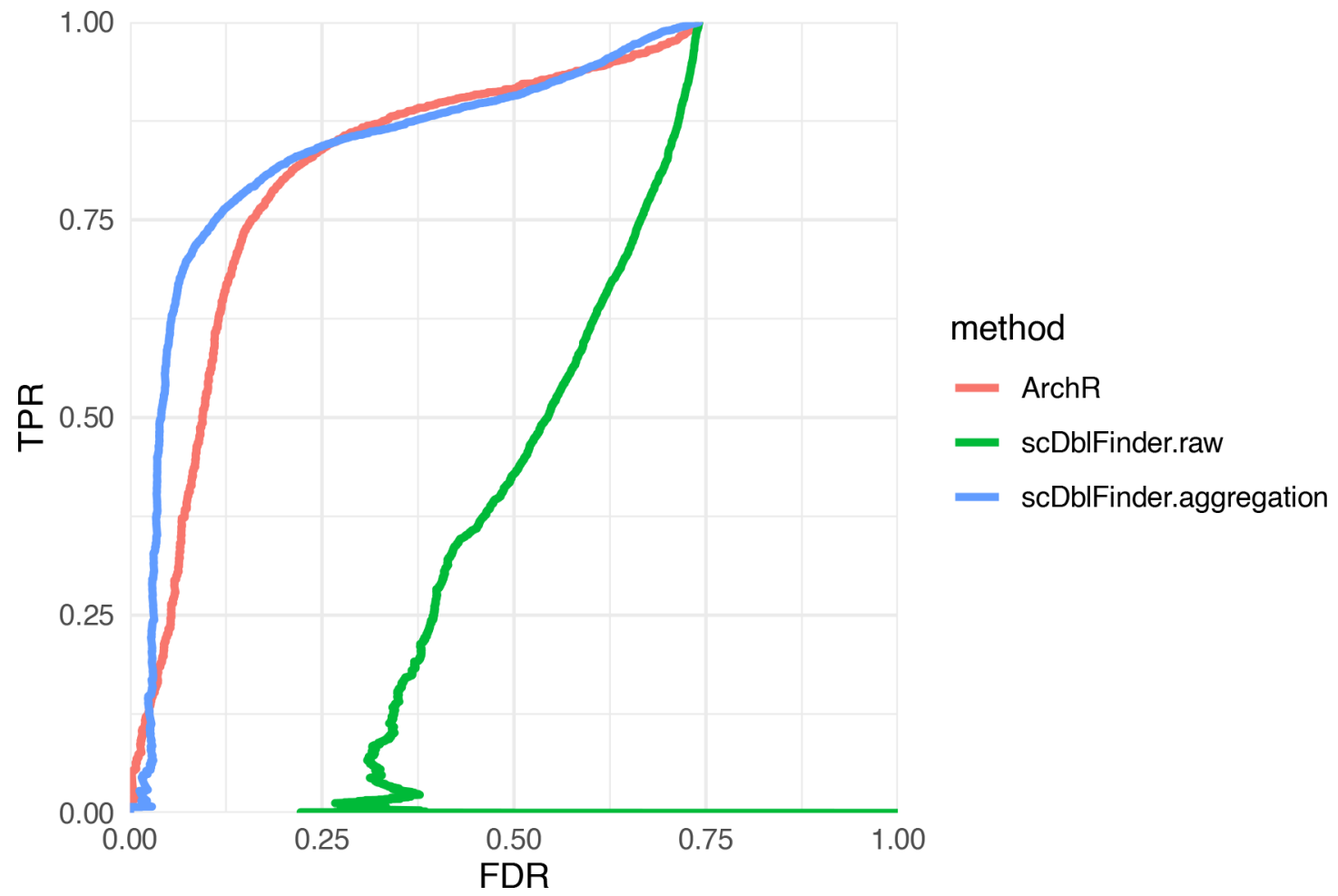

Figure 8. Doublet identification in single-nucleus ATAC-seq. Performance of scDblFinder with default (.raw) parameters or on aggregated features (.aggregation) versus ArchR (GSE162690 dataset).

performed and the features are clustered into the desired number of meta-features using mini-batch k-means (Hicks et al. 2021) or, if not available, simple k-means. The counts are then summed per meta-feature.

\section{scDblFinder operation}

$s c D b l F i n d e r$ is provided as a bioconductor package. The input data for scDblFinder (denoted $x$ below) can be either i) a count matrix (full or sparse), with genes/features as rows and cells/droplets as columns; or ii) an object of class 


\section{A: Expected doublet proportions}

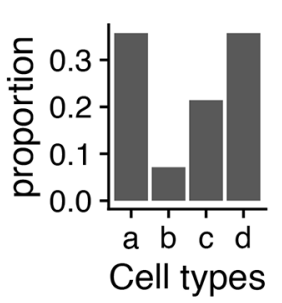

B: Enrichment scenarios 'Sticky' Enriched cell type combination fold-

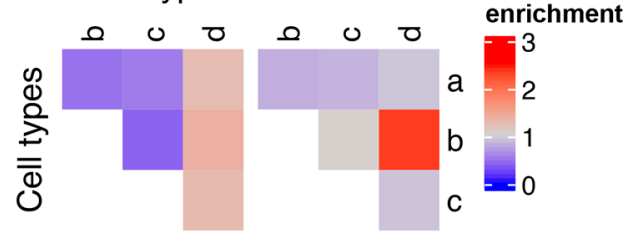

Cell types
C

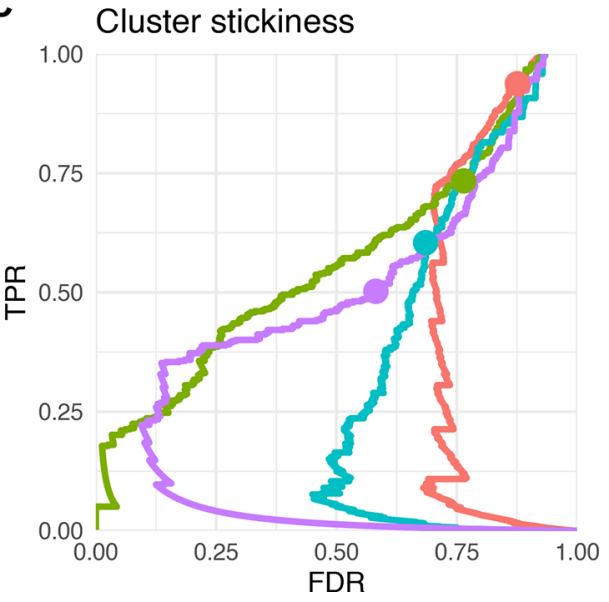

D Specific combinations

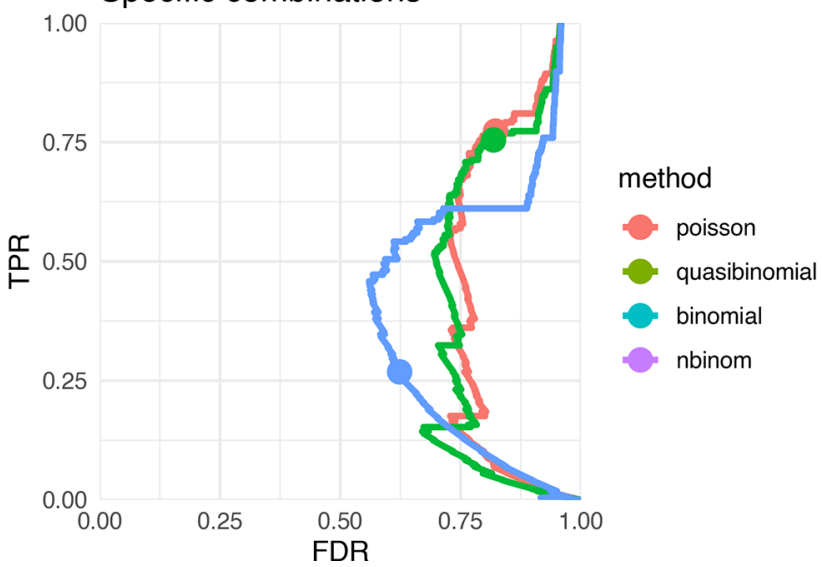

Figure 9. Doublet enrichment analysis. A, B: Doublet enrichment in a toy example. A: Proportion of different doublet types from random expectations based on the cell type abundances. B: The fold-enrichment over this expectation in two different doublet enrichment scenarios. C, D: Performance of the cluster stickiness tests (C) and tests for enrichment of specific combinations (D) using different underlying distributions.

SingleCellExperiment. In either case, the object should not contain empty drops, but should not otherwise have undergone very stringent filtering (which would bias the estimate of the doublet rate). The doublet detection can then be launched with:

library (scDblFinder)

sce $<-\operatorname{scDblFinder}(x)$

The output is a SingleCellExperiment object including all of the input data, as well as a number of columns to the colData slot, the most important of which are:

- sce\$scDblFinder.score: the final doublet score (the higher the more likely that the cell is a doublet)

- sce\$scDblFinder.class: the binary classification (doublet or singlet)

scDblFinder can run on any system running $\mathrm{R}>=4.0$ and Bioconductor $>=3.12$.

For more details, see the package's vignettes.

\section{Results}

Characterization of real doublets

As most approaches rely on some comparison of real cells to artificial doublets, it is crucial to appropriately simulate doublets. To this end, we first characterized real doublets using a dataset of genetically distinct cell lines (Tian et al. 2018). Because each cell line represents a distinct and more or less homogeneous transcriptional state, it is possible to identify the 'cell types' composing each doublet (Figure 3). Although often larger, the median library sizes of doublets were 
systematically smaller than the sum of the median library sizes of composing cell types (Figure 3A). We next investigated the relative contributions of the composing cell types using non-negative least square regression, expecting the larger cell types to contribute more to the doublet's transcriptome.

Although differences in median library size across cell types were small (less than two-fold) compared to other datasets, we observed a weak association of the relative contributions with the relative sizes of the composing cell types (Figure 3B, $p=2 \mathrm{e}-10)$. This effect was however considerably smaller than the variation within doublet type. This suggests that there are i) large variations in real cell size within a given cell type, and/or ii) large variations in the mRNA sampling efficiency that are independent for the two composing cells. In light of these ambiguities, we opted for a mixed strategy in the generation of artificial doublets: a proportion is generated by summing the libraries of individual cells, another by performing a poisson resampling of the obtained counts, and a third by re-weighting the contributions of cells depending on the relative median sizes of the composing cell types.

\section{scDblFinder outperforms alternative methods}

A previous version of scDblFinder was already compared, and shown to be superior to existing alternatives in an independent benchmark by Xi and $\mathrm{Li}$ (2021a). Here we reproduced this benchmark using the most recent versions of the packages, and including variant methods from the scDblFinder package (among which the updated version of scran's original method, and now available in the scDblFinder package as computeDoubletDensity). Figure 4 compares the performance of $s c D b l F i n d e r$ to alternatives across the real benchmark datasets. scDblFinder has the highest mean area under the precision-recall (PR) curve (see Germain 2021b, Figure 7), ranking first in a majority of datasets, and otherwise typically very close to the top. In addition, scDblFinder runs at a fraction of the time required by the next best methods (Figure 4, left).

\section{Most heterotypic doublets are accurately identified}

Several of the benchmark datasets have true doublets flagged by their mixing of single-nucleotide polymorphisms from multiple individuals (Kang et al. 2018). In most of these cases, however, the doublets include also inter-individual homotypic doublets (in the sense of being a combination of cells of the same type from different individuals), which are difficult to detect from gene expression (Figure 5A). In addition, they miss heterotypic doublets that are the result of the combination of different cell types from the same individual. Indeed, datasets where there is a full correspondence between cell type and individual (such as the human-mouse mixtures, e.g. hm-6k and hm-12k) typically have a much higher area under the Receiver-operator characteristic (ROC) and precision-recall (PR) curves (Figure 4). It is therefore likely that the accuracy reported in the benchmark is below the actual one in detecting heterotypic doublets. Based on the frequency of the different individuals and cell types in a dataset, it is possible to infer the expected rate of inter-individual homotypic doublets and within-individual heterotypic doublets. This, in turns, allows us to adjust the measured true positive rate (TPR) and false discovery rate and get a better picture of our ability to detect heterotypic doublets. Figure 5B shows such an analysis for a complex dataset from Kang et al. (2018). The inflection point of the PR curve roughly coincides with the expected proportion of heterotypic doublets among those flagged as true doublets.

Adjusting for both types of error in the truth, the area under the PR curve is considerably better ( 0.82 instead of 0.64$)$, and at the automatic threshold we estimate that $87 \%$ of heterotypic doublets can be identified with a real FDR of $32 \%$ (a similar analysis for a different sample is shown in Germain 2021b, Figure 9).

\section{Flexible thresholding for doublet calling}

Most doublet detection methods provide a 'doublet score' that is higher in doublets than in singlets, and users are left to decide on a threshold above which cells will be excluded as doublets. Because scDblFinder's scores come from a classifier, they can directly be interpreted as a probability. Nevertheless, a threshold needs to be set, and it should ideally be placed at the inflection point (assuming there is one) of the ROC or PR curve, so that most doublets and not too many singlets are excluded. While these curves are typically not available in practice, we found that in most cases the $s c D b l$ Finder scores are rapidly changing from high to low very close to the inflection point (Figure 6). One possibility is therefore to use directly a fixed probability threshold to call doublets. In some cases, however, there is a more gradual change in score (e.g. nuc-MULTI dataset), making it more difficult to establish a threshold in a non-arbitrary fashion. Building on the fairly tight relationship (especially in 10x-based datasets) between the number of cells captured and the rate of doublets generated (Kang et al. 2018), another approach consists in setting the threshold based on the number of doublets (or heterotypic doublets) one expects to find in the data. scDblFinder includes a thresholding method that combines both rationales, and attempts to minimize both the proportion of artificial doublets being misclassified and the deviation from the expected doublet rate (see Thresholding).

The identified thresholds are shown in Figure 6A-B, and compared to thresholds based on the expected doublet rate in Figure 6C. In general, scDblFinder thresholds are closer to the inflection point. 


\section{Doublet detection across multiple samples/captures}

Multiple samples are often profiled and analyzed together, with the very common risk of batch effects (either technical or biological) across samples (Lütge et al. 2021). Therefore, while the cells from all samples might in principle provide more information for doublet detection than a single sample can afford on its own, this must be weighted against the risk of bias due to technical differences. To investigate this, we implemented different multi-sample approaches and tested them on two real multi-sample datasets with demuxlet-based true doublets, as well as a sub-sampling of them (Figure 7).

The different multi-sample strategies had only a minor impact on the accuracy of the identification. Based on these results, the best overall strategy appears to be to process all samples as if they were one, however in our experience this can lead to biases against some samples when there are very large variations (e.g. in number of cells or coverage) across samples (not shown). This approach also greatly increases running time. In contrast, running the samples fully separately is computationally highly efficient, and is often equally accurate.

\section{SCATACseq: aggregating rather than selecting features}

We next investigated whether scDblFinder could be applied to other types of single-cell data prone to doublets, such as single-cell Assay for Transposase-Accessible Chromatin sequencing (ATACseq). To evaluate this, we used the mixture of 10 cell lines from Granja et al. (2021). With default parameters, scDblFinder performed very poorly (Figure 8). This is chiefly because scDblFinder follows the common scRNAseq strategy of selecting an informative subset of the features, while ATACseq reads are typically sparsely distributed across the genome. However, working with all features (i.e. peaks) is computationally very expensive. An alternative to both approaches is to begin by reducing the size of the dataset by aggregating correlated features into a relatively small set, thereby using information from all. These aggregated features can then directly be used as the space in which to calculate distances. This method yielded equal or better performance than specialized single-cell ATACseq software (Figure 8).

\section{Doublet origins and enrichment analysis}

When artificial doublets are generated between clusters, we can keep track of the clusters composing them, and we reasoned that this information could be used to infer the clusters composing real doublets (hence after referred to as 'doublet origin'). Using a simulation as well as the aforementioned real dataset with doublets of known origins (mixture of five cell lines from Tian et al. (2018)), we assessed the accuracy of doublet origin prediction based on the nearest artificial doublets in the kNN. These proved inaccurate, both in real and simulated data (see Germain 2021b, Figure 9A-B). Even training a classifier directly on this problem failed (see Germain 2021b, Figure 9C-D). The problem appears to be that, due to the very large variations in library sizes (and related variations in relative contributions of the composing cells - see Figure 3B), doublets often contain a large fraction of reads from one cell type, and conversely a small fraction from the other cell type. As a consequence, we can typically call at least one of the two originating cell types, but seldom both. In the real dataset, at least one of the two originating cell type is correctly identified in $73 \%$ of doublets (random expectation: $36 \%$ ), but both are correct in only $20 \%$ of cases.

While the identification of doublet origins remains a challenge, for the sake of completeness we nevertheless developed strategies to investigate whether certain doublet types were found more often than expected. Such enrichment could, for instance, indicate cell-to-cell interactions. We defined two forms of doublet enrichment (Figure 9A-B), and specified models to test each possibility: i) enrichment in doublets formed by a specific combination of celltypes, or ii) enrichment in doublets involving a given cell type, denoted 'sticky.'

The 'stickiness' of each cluster (as proxy for cell types) can be evaluated by fitting a single generalized linear model on the observed abundance of doublets of each origin (see Methods). We tested the performance of this test under different underlying distributions using simulated doublet counts. The number of doublets of each type is generated from random expectation with or without added stickiness (as factors of 1 to 3 on the probability) using negative binomial distributions with different over-dispersion parameters (Figure 9C and Germain 2021b, Figure 10). The quasi-binomial showed the best performance, followed by the negative binomial, but in all cases the $\mathrm{p}$-values were not well calibrated and many false positives were reported at a nominal FDR $<0.05$. This was robust across different over-dispersion values (see Germain 2021b, Figure 10).

We next sought to establish a test for the enrichment of specific combinations. Here, we simply computed the probability of the observed counts for each combination using different models (see Methods). We again tested this approach relying on different underlying distributions, on simulations with varying over-dispersion. The negative binomial performed best, however all variants suffered a high false discovery rate (Figure 9C). 


\section{Conclusions}

The characterization of real doublets suggests a multi-layered variation in mRNA capture efficiency, and calls for a varied approach to artificial doublet generation. The scDblFinder package includes a set of efficient methods for doublet detection. In particular, the main $s c D b l F i n d e r$ approach uses mixed doublet generation approaches and integrates insights from previous approaches into a comprehensive doublet detection method that provides robustly accurate detection across a number of benchmark datasets, at a considerably greater speed and scalability than the best alternatives. Even in complex datasets, most heterotypic doublets can be accurately identified. Although the doublet scores given by $s c D b l$ Finder can be directly interpreted as probabilities, simplifying their interpretation, the method also includes a tradeoff thresholding procedure incorporating doublet rate expectations with classification optimization, thereby facilitating its usage. Finally, we further demonstrate that, with slight changes in parameters, the approach is also amenable to other data types such as single-nucleus ATAC-seq.

scDblFinder additionally provides utilities for identifying the origins of doublets (in terms of composing cell types) and testing for different forms of doublet enrichment. At present, however, the value of such tests is limited by the difficulty of accurately identifying doublet origins. Further research will be needed to assess to what extent this can be improved.

In conclusion, we believe that scDblFinder, with its flexibility, accuracy and scalability, represents a key resource for doublet detection in high-throughput single-cell sequencing data.

\section{Software availability}

scDblFinder is available from Bioconductor: http://www.bioconductor.org/packages/release/bioc/html/scDblFinder.html.

The source code is available from: https://github.com/plger/scDblFinder.

Archived source code at time of publication: https://doi.org/10.6084/m9.figshare.16543518.v1 (Germain, 2021a).

The software is released under the GNU Public License (GPL-3).

\section{Data availability}

Underlying data

figshare: scDblFinder. https://doi.org/10.6084/m9.figshare.16543518.v1 (Germain, 2021a).

This repository contains the following underlying data:

- $\quad$ scDblFinder 1.7.4 (archived software version used in the paper).

- scDblFinder_paper (code to reproduce the analyses and figures).

The code to reproduce the analyses and figures is additionally available at https://github.com/plger/scDblFinder_paper.

Data are available under the terms of the Creative Commons Attribution 4.0 International license (CC-BY 4.0).

Extended data

figshare: Supplementary Figures for the scDblFinder paper. https://doi.org/10.6084/m9.figshare.16617571.v1 (Germain, 2021b)

This repository contains the following extended data:

- Supplementary Figures 1-10

Data are available under the terms of the Creative Commons Attribution 4.0 International license (CC-BY 4.0).

\section{Acknowledgments}

We thank Nan Miles Xi and Jingyi Jessica Li for help with their benchmark datasets; Jeffrey Granja for support with ArchR and its attached dataset; and the Robinson group and users for feedback. MDR acknowledges support from the University Research Priority Program Evolution in Action at the University of Zurich. 
Amezquita RA, Lun ATL, Becht E, et al.: Orchestrating Single-Cell Analysis with Bioconductor. Nat. Methods. December, 1-9 2019; 17: 137-145. Publisher Full Text

Bais AS, Kostka D: Scds: Computational Annotation of Doublets in Single-Cell RNA Sequencing Data. Bioinformatics. 2020; 36(4): 1150-58. PubMed Abstract | Publisher Full Text | Free Full Text

Bernstein NJ, Fong NL, Lam I, et al.: Solo: Doublet Identification in Single-Cell RNA-Seq via Semi-Supervised Deep Learning. Cell Systems. 2020 June; 11: 95-101.e5.

PubMed Abstract | Publisher Full Text

Bloom JD: Estimating the Frequency of Multiplets in Single-Cell RNA Sequencing from Cell-Mixing Experiments. Peer). 2018; 6(September) e5578.

PubMed Abstract | Publisher Full Text | Free Full Text

DePasquale EAK, Schnell DJ, Van Camp P-J, et al.: DoubletDecon:

Deconvoluting Doublets from Single-Cell RNA-Sequencing Data.

Cell Rep. 2019; 29(6): 1718-1727.e8.

PubMed Abstract | Publisher Full Text | Free Full Text

Germain, Pierre-Luc: scDbIFinder. figshare. Software. 2021 a.

Publisher Full Text

Germain, Pierre-Luc: Supplementary Figures for the scDblFinder paper. figshare. Figure. 2021b.

Reference Source

Germain P-L, Sonrel A, Robinson MD: pipeComp, a General Framework

for the Evaluation of Computational Pipelines, Reveals Performant

Single Cell RNA-Seq Preprocessing Tools. Genome Biol. 2020; 21(1): 227.

PubMed Abstract | Publisher Full Text | Free Full Text

Granja JM, Ryan Corces M, Pierce SE, et al.: ArchR Is a Scalable Software Package for Integrative Single-Cell Chromatin Accessibility Analysis. Nat. Genet. 2021 February, 1-9; 53: 403-411.

Publisher Full Text

Hicks SC, Liu R, Ni Y, et al.: Mbkmeans: Fast Clustering for Single Cell Data Using Mini-Batch k-Means. PLoS Comput Biol. 2021: 17(1): e1008625.

PubMed Abstract | Publisher Full Text | Free Full Text
Kang HM, Subramaniam M, Targ S, et al.: Multiplexed Droplet Single-Cell RNA-Sequencing Using Natural Genetic Variation. Nat. Biotechnol. 2018; 36(1): 89-94.

PubMed Abstract | Publisher Full Text | Free Full Text

Lütge A, Zyprych-Walczak J, Kunzmann UB, et al.: CellMixS: Quantifying and Visualizing Batch Effects in Single-Cell RNA-Seq Data. Life Sci. Alliance. 2021; 4(6): e202001004.

PubMed Abstract | Publisher Full Text | Free Full Text

McGinnis CS, Murrow LM, Gartner ZJ: DoubletFinder: Doublet Detection in Single-Cell RNA Sequencing Data Using Artificial Nearest

Neighbors. Cell Systems. 2019; 8(4): 329-337.e4.

PubMed Abstract | Publisher Full Text | Free Full Text

Stoeckius M, Zheng S, Houck-Loomis B, et al.: Cell Hashing with Barcoded Antibodies Enables Multiplexing and Doublet Detection for Single Cell Genomics. Genome Biol. 2018; 19(1): 224.

PubMed Abstract | Publisher Full Text | Free Full Text

Stuart T, Butler A, Hoffman P, et al.: Comprehensive Integration of Single-Cell Data. Cell. 2019; 177(7): 1888-1902.e21.

PubMed Abstract | Publisher Full Text | Free Full Text

Tian L, Dong X, Freytag S, et al.: scRNA-Seq Mixology: Towards Better Benchmarking of Single Cell RNA-Seq Protocols and Analysis

Methods. bioRxiv. 2018 October.

Publisher Full Text

Wolock SL, Lopez R, Klein AM: Scrublet: Computational Identification of Cell Doublets in Single-Cell Transcriptomic Data. Cell Systems. 2019; 8(4): 281-291.e9.

PubMed Abstract | Publisher Full Text | Free Full Text

Xi NM, Li J]: Benchmarking Computational Doublet-Detection Methods for Single-Cell RNA Sequencing Data. Cell Systems. 2021a; 12(2): 176-194. e6.

PubMed Abstract | Publisher Full Text | Free Full Text

$\mathrm{Xi} \mathrm{NM}, \mathrm{Li} \mathrm{J}$ : Protocol for Executing and Benchmarking Eight Computational Doublet-Detection Methods in Single-Cell RNA Sequencing Data Analysis. arXiv:2101.08860 [q-Bio]. 2021b June.

Reference Source 


\section{Open Peer Review}

\section{Current Peer Review Status:}

\section{Version 1}

Reviewer Report 08 November 2021

https://doi.org/10.5256/f1000research.77262.r95560

(C) 2021 Kostka D et al. This is an open access peer review report distributed under the terms of the Creative Commons Attribution License, which permits unrestricted use, distribution, and reproduction in any medium, provided the original work is properly cited.

\section{Dennis Kostka}

Department of Developmental Biology; Department of Computational \& Systems Biology; Pittsburgh Center for Evolutionary Biology and Medicine, University of Pittsburgh School of Medicine, Pittsburgh, PA, USA

\section{Hannah Schriever}

${ }^{1}$ Department of Developmental Biology, University of Pittsburgh School of Medicine, Pittsburgh, PA, USA

${ }^{2}$ Joint CMU-Pitt Ph.D. Program in Computational Biology, Pittsburgh, PA, USA

\section{Summary}

The authors describe scDblFinder, a computational tool for doublet/multiplet annotation in singlecell RNA sequencing and single-cell ATAC sequencing data. They explain and motivate method design and parameter choices, reproduce a benchmark on scRNA-seq data (originally performed by Xi and Li 2021) and demonstrate scDblFinder's performance; this includes inferring cell-types of origin for doublets, deriving binarized doublet calls, and annotating doublets SCATAC-seq data.

\section{General Comments}

The reviewers note that they have not considered other reviews for the same manuscript prior to or during the writing of this report.

scDblFinder is probably the most competitive method for computational doublet annotation, so this manuscript, describing the method in detail, is an important contribution and valuable for the community. We have the following general comments:

Positive points of the manuscript that stand out are the authors' discussion of their results on annotating cell-types of origin for doublets (and the conclusion that it remains a challenge), scDblFinder's good performance on scATAC-seq data, and its thresholding approach for generating binarized doublet calls.

While the quality of the manuscript is generally high, we still feel that clarity can be 
improved, especially in describing methods (see specific comments).

Further on, as the authors describe, parameters of scDblFinder have been tuned to maximize performance on 16 benchmark data sets used for evaluation. As a consequence, performance comparison with methods that did not perform parameter optimization on the benchmark data is biased in favor of scDblFinder; this should be mentioned in the manuscript's discussion section.

\section{Specific Comments}

Terminology: It would be good to consistently use a term (like "droplet", for example) for sequenced units of RNA that can contain more than one cell, whereas the term "cell" could be reserved to indicate a single cell.

In the introduction, the authors comment on drawbacks of multiplexing technologies and that they only identify a fraction of doublets. It would be good to (a) include that these experimental methods incur additional drawbacks, like increased cost and decreased yield and (b) that there are definite benefits of sample multiplexing in the context of experimental design.

Methods, page 3: It would be helpful to streamline the description of scDblFinder's approach/procedure. Specifically, a verbal description would help. In the current version, for example, the authors talk about "the cluster-based approach", but it is not at all clear what they mean at that point in the manuscript.

Methods, page 4: The authors state that mislabeled cells can mislead the classifier and use this to motivate their iterative approach (in paragraph 2). It would be helpful to reference Supplemental figure 5 here, which shows the positive effect of removing doublet-classified but singlet-annotated droplets.

Formula on page 5: It is not discussed how the $d b r . s d$ parameter that is used for $e_{-} l o w$ and e_high is estimated for a given dataset. Is there a motivation for choosing this specific functional form for deviation?

Figure 2: It looks like the dashed line is not at the minimum of the cost function (black line).

Formulae on page 6 and 8: It is not described how difficulty is estimated from misclassification of simulated doublets. In general, it would be good to present a consistent discussion for both models, including motivation, definition of all quantities, and error terms.

For the dataset used in Figure 3, it would be helpful to give a more in detailed description of the cell lines (organism, type, etc).

Methods page 9. In paragraph 1, how is the desired number of features determined (is it a method parameter)?

Results page 10. It would be helpful to briefly describe cell lines in the Tian et al. 2018 dataset (see previous comment about Figure 3). 
Results page 11. It would be helpful to add details about how artificial doublets were generated in paragraph 2. How were contributions "re-weighted based on the relative median sizes of the composing cell-types"?

Results page 11. In paragraph 4 and Figure 5 panel B: How was the expected rate of homotypic / heterotypic doublets estimated? In the legend of Figure 5, maybe change "truth" to "annotation" (also in the short paragraph 5 on page 11). Further on, a description on how the adjusted AUPRC is calculated should be included. Since doublet prediction is imbalanced, has a non-zero minimum AUPRC (https://dl.acm.org/doi/10.5555/3042573.3042780 ${ }^{1}$ ) been taken into account as well? Finally, does the black dot in Figure $5 \mathrm{~B}$ denote a cutoff such that the number of doublets scDblFinder would call corresponds to the expected number of heterotypic doublets?

Results page 11. In paragraph 5 (3 lines), it would be helpful to make explicit what two types errors are accounted for. Further on, it appears the adjustment is based on estimated doublet rates, and it would be good to discuss how reliable these are, and how that, in turn, impacts the adjusted areas under the curve. Finally, the reference should be to supplemental figure 8 (not 9).

Figure 6: It is not clear what the dots in panel A denote. In addition, it is really hard to compare the different points across panels B and C; perhaps sub-panels (one per curve) with both points on each curve would be clearer.

Results page 11. For the last paragraph, it would be good to provide metrics that compare the two approaches in a qualitative way. One possibility would be a table showing for each benchmark dataset the number of annotated doublets, called doublets, false positives and false negatives together with a performance metric (e.g., Matthews correlation coefficient).

Results, page 12. The authors conclude from Figure 7 that "as one" is the "best overall strategy". Performance seems really close (and the conclusion only holds for AUPRC, not really for AUROC), so it is not clear if (a) the advantage is significant and (b) is expected to generalize to other data sets. This could be discussed.

Figure 8 is very large.

Figure 9: Some colors are different between panels $C$ and $D$, which makes interpretation of the legend confusing.

Supplemental Figure 1: It would be helpful to better explain the setup in the legend, so it is clear what TRUE/FALSE mean and how differences in AUPRC are defined (e.g., high = better performance without variable, low = worse performance without variable). If the violins are summarizing 16 points, it might be informative to show the points themselves. Does the dot indicate the median or the mean, what interval is denoted?

Supplemental Figure 2: In panel B, maybe show log of elapsed time.

Supplemental Figure 3: Consider using the same type of violin plot as before. Typo on page 5: "based on the given or estimated doublet rate the dbr.sd parameter" should be "based on the given or estimated doublet rate and the dbr.sd parameter". 
Typo on page 8: "probability of each double type" should be " probability of each doublet type

Type on page 11: "This, in turns, allows" should be "This, in turn, allows"

\section{References}

1. Boyd K, Santos Costa, Davis J, David Page: Unachievable region in precision-recall space and its effect on empirical evaluation. Omnipress. 2012. 1619-1626 Reference Source

Is the rationale for developing the new software tool clearly explained?

Yes

Is the description of the software tool technically sound?

Yes

Are sufficient details of the code, methods and analysis (if applicable) provided to allow replication of the software development and its use by others?

Yes

Is sufficient information provided to allow interpretation of the expected output datasets and any results generated using the tool?

Yes

Are the conclusions about the tool and its performance adequately supported by the findings presented in the article?

Yes

Competing Interests: No competing interests were disclosed.

Reviewer Expertise: computational genomics, evolutionary genomics, statistical phylogenetics

We confirm that we have read this submission and believe that we have an appropriate level of expertise to confirm that it is of an acceptable scientific standard.

Author Response 23 Apr 2022

Pierre-Luc Germain

We thank the reviewers for their thorough reading and useful comments, and have tried to address all of them in the revised version. A detailed point-by-point version will be made available along with the new version, but here are the main points of response:

1. Bias due to parameter optimization based on the benchmark datasets: The reviewers were absolutely right to raise this important point, we now state it clearly in the discussion, with the note however that a previous version of scDblFinder (developed with only 5 of the datasets) was also found to outperform alternatives in 
the addendum to Xi and Li's (2020) original benchmark.

2. Terminology and clarifications: We thank the reviewers for their excellent suggestions regarding terminology and clarifications, which we adopted in the revised version (see the full point-by-point response for details). We also tried to provide a simpler 'verbal' overview of the method in the Results section, as suggested, and to clarify the methods section.

3. AUPRC: We thanks the reviewer for the suggested paper on AUPRC. While it was quite an interesting read, we do not think that either the random or the minimal AUPRC affects any of the claims being made, as it is certainly well below any of those observed, and our main point in this section is that the performance in estimating heterotypic doublets is underestimated in most of the benchmark datasets. This being said, we now added the random expectation in Figure 4B.

4. Finally we also addressed the minor comments.

Competing Interests: No competing interests were disclosed.

Reviewer Report 19 October 2021

https://doi.org/10.5256/f1000research.77262.r95679

(c) 2021 Gartner $Z$ et al. This is an open access peer review report distributed under the terms of the Creative Commons Attribution License, which permits unrestricted use, distribution, and reproduction in any medium, provided the original work is properly cited.

Zev J. Gartner

Department of Pharmaceutical Chemistry, University of California, San Francisco, San Francisco, CA, USA

\section{Chris McGinnis}

University of California, San Francisco, CA, USA

\section{Summary}

The authors describe scDblFinder - a doublet detection algorithm that uses gradient boosted trees to classify doublets as cells with elevated proportions of artificial nearest neighbors, library sizes, and co-expression scores. The authors describe parameter selection and hyperparameter optimization workflows before benchmark their method against the Xi \& Li datasets. These efforts effectively demonstrate that scDblFinder performs very strongly relative to other doublet detection methods. Moreover, building on the existing doublet detection literature, the authors also make the following improvements/alterations to existing workflows and reaffirmations of previous observations:

Authors simulate doublets using a 'mixed strategy' including summing counts, Poisson resampling of counts, and weighted-averaging of counts based on cell-type-specific library 
sizes. This approach is distinct from current methods which simulate doublets by summing/averaging counts or averaging PC coordinates - Perhaps an improvement but needs more analysis (Major comment \#1).

Authors demonstrate that scDblFinder is predominantly sensitive to heterotypic doublets and that removing homotypic doublets from ground-truth data improves doublet prediction performance metrics - Affirming previous observations in the DoubletFinder and other manuscripts.

Authors threshold doublet classification probability distributions (i.e., likelihood scores generated from gradient boosting) using a combination of two known approaches: (i) Threshold based on artificial doublet misclassification rate (from Scrublet) or (ii) Threshold based on expected number of doublets inferred from droplet microfluidics Poisson loading statistics (from DoubletFinder) - Perhaps an improvement (Major comment \#2).

Authors show that scDblFinder (and other classifiers) should be run on individual SCRNA-seq samples in instances when those samples are not multiplexed using Cell Hashing, MULTIseq, etc. - This is an 'unwritten' rule for doublet detection workflows but hadn't been definitively demonstrated.

Authors show that scDblFinder performs as well as ArchR for predicting doublets in snATACseq data after aggregating features - This is good to know, but authors need to include the current gold-standard for snATAC-seq doublet detection in their analysis, AMULET, such that readers are correctly informed about their analytical options for snATAC-seq doublet detection (Major Comment \#3).

Authors present a strategy for inferring doublet origins which could be useful for identifying instances of biological doublets formed due to cell-cell interactions or 'stickiness' - Could be useful theoretically, but the approach is not sufficiently developed to be useful (although we commend the authors for including this analysis)

On balance, we believe that scDblFinder is a great method (likely the new gold standard for the field) and strongly recommend its indexing, assuming that our Major Comments are addressed.

\section{Major Comments}

1. The authors do a good job at justifying the theoretical utility of the proposed 'mixing strategy' for doublet simulation in Figure 3. However, while the authors compare scDblFinder performance before/after dimensionality reduction and kNN and using clusterlimited vs random doublet simulation, they do not do analogous analyses for comparing the 'mixing strategy' to more standard methods (e.g., summing, averaging, etc.). As such, it is unclear whether the proposed strategy improves performance.

2. In Figure 6, the authors plot ROC curves annotated by the thresholds defined by their proposed cost-minimization framework (Fig. 6B) or by the expected number of doublets (Fig. 6C). Since the cost-minimization framework also aims to minimize artificial doublet misclassification rate (as implemented by Scrublet), we would also like to see the same ROC plots with thresholds defined by this strategy (i.e., instead of just the expected doublet 
strategy implemented by DoubletFinder). We would also like the authors to provide summary statistics for the comparisons (see Minor Comment \#1) Without this, it is unclear whether the cost-minimization framework is actually an improvement over existing approaches.

3. The application of scDblFinder to snATAC-seq data is not satisfactory as it only involves benchmarking against ArchR, which was recently shown in the AMULET paper (Thibodeau, Eroglu, et al. Genome Biology, 2021) to perform sub-optimally on snATAC-seq data. The authors need to include reference to AMULET in this section and benchmark the performance of AMULET, ArchR, and scDblFinder.

4. We believe that the manuscript in its current form suffers from poor readability - we really had to parse every sentence in the Methods section to understand what the authors were intending to convey. For one tangible example, when discussing the overfitting hypothesis on page 4 (which itself was conveyed quite clearly), the authors could include a clear rationale for why comparing scDblFinder performance before and after dimensionality reduction and kNN addresses the hypothesis. Notably, we believe that this manuscript was very well-executed and that scDblFinder is a great method. We also recognize that the authors write very accessibly in the Results section. However, many in the single-cell genomics community who may want to use scDblFinder are not machine learning experts will likely have a hard time appreciating the quality of this work, especially in its current structure (i.e., Methods section before Results). As such, we strongly recommend that the authors add more 'plain English' explanations to the Methods section (or put the Results before the Methods section, if the journal will allow that) to improve readability for a broader audience.

\section{Minor Comments}

1. Should provide quantitative analysis of distance of scDblFinder vs heterotypic doublet thresholding strategies to the real inflection point (related to Figure 6).

\section{Authors should cite papers related to the benchmarking datasets used in Xi \& Li. ${ }^{1}$}

3. Authors should cite Chord from Xiong and colleagues, which also uses boosting to improve doublet detection. ${ }^{2}$

4. On page 3, the authors state that multiplexing approaches "identify only a fraction of the doublets" - this is technically true, but a bit misleading. The fraction of doublets detected in multiplexing experiments scales with the number of multiplexed samples. In instances where only a handful of samples are multiplexed, it is fair to say that 'only a fraction' are identified. However, any modestly large experiment will result in the vast majority of doublets being detected. And even in the lowly multiplexed contexts, it is straightforward to remove heterotypic at the cluster-level by identifying clusters enriched for empiricallydefined doublets. Authors should clarify (or remove) this statement as it suggests that scDblFinder is a 'competitor' with multiplexing approaches for doublet detection performance. Computational prediction will very rarely out-compete empirical measurements - instead, scDblFinder has the key advantage of being applicable to any past or future scRNA-seq data, which is not the case for Cell Hashing, MULTI-seq, etc. 


\section{References}

1. Xi N, Li J: Benchmarking Computational Doublet-Detection Methods for Single-Cell RNA Sequencing Data. Cell Systems. 2021; 12 (2): 176-194.e6 Publisher Full Text

2. Xiong K, Zhou H, Yin J, Kristiansen K, et al.: Chord: Identifying Doublets in Single-Cell RNA Sequencing Data by an Ensemble Machine Learning Algorithm. bioRxiv. 2021. Publisher Full Text

Is the rationale for developing the new software tool clearly explained?

Yes

Is the description of the software tool technically sound?

Yes

Are sufficient details of the code, methods and analysis (if applicable) provided to allow replication of the software development and its use by others?

Yes

Is sufficient information provided to allow interpretation of the expected output datasets and any results generated using the tool?

Yes

Are the conclusions about the tool and its performance adequately supported by the findings presented in the article?

Yes

Competing Interests: No competing interests were disclosed.

Reviewer Expertise: Tissue engineering and single cell analysis method development

We confirm that we have read this submission and believe that we have an appropriate level of expertise to confirm that it is of an acceptable scientific standard, however we have significant reservations, as outlined above.

Author Response 23 Apr 2022

Pierre-Luc Germain

We thank the reviewers for their thorough reading and useful comments, and have tried to address all of them in the revised version. A detailed point-by-point version will be made available along with the new version, but here are the main points of response:

1. Artificial doublet generation: The reviewers are right that we simply assumed, because the doublets showed lower library size than expected from summation, that the mixed strategy would be superior. We now tested this, along with averaging. While averaging expectedly resulted in poorer performance, we in fact did not see a clear overall improvement of the mixed strategy. We now discuss this explicitly.

2. Thresholding: Following the reviewers' suggestions, we also rewrote much of this section in a more open fashion, reflecting the choices that ultimately enter any 
thresholding decision (i.e. the lack of an objective optimal threshold), and changed the figure to provide clearer readouts that compare more alternative procedures.

3. scATACseq: Thanks for the suggestions, at the time of submission we were not aware of the AMULET method. We now included it in the comparison, along with the dataset with ground truth with which it was published. We also reimplemented the method in our package, for the convenience of $\mathrm{R}$ users.

4. Text structure and clarity: With permission from f1000, we now moved the Methods section to the end (as it was originally conceived), tried to offer a simpler overview of the method in the Results section, and to clarify the methods.

5. Finally we also addressed the minor comments.

Competing Interests: No competing interests were disclosed.

The benefits of publishing with F1000Research:

- Your article is published within days, with no editorial bias

- You can publish traditional articles, null/negative results, case reports, data notes and more

- The peer review process is transparent and collaborative

- Your article is indexed in PubMed after passing peer review

- Dedicated customer support at every stage

For pre-submission enquiries, contact research@f1000.com 